\title{
Fidelidad al sitio y rango de hogar del caballito de mar narizón Hippocampus reidi (Teleostei: Syngnathidae) en la dársena de Varadero, noroeste de Cuba
}

\author{
Site fidelity and home range of the longsnout seahorse \\ Hippocampus reidi (Teleostei: Syngnathidae) at the Varadero \\ dock, northwest Cuba
}

\author{
Daril de la Nuez Hernández ${ }^{1 *}$, Lourdes Pastor Gutiérrez ${ }^{1}$, Alejandro Pérez Angulo ${ }^{1}$, Yuliet \\ Piloto Cubero ${ }^{1}$ y Raúl Igor Corrada Wong ${ }^{1}$
}

\begin{abstract}
RESUMEN
En las últimas décadas se ha constatado un importante declive mundial en las poblaciones de peces de la familia Syngnathidae, entre las que se encuentran los carismáticos caballitos de mar. Hasta el momento en Cuba han sido escasas las investigaciones realizadas a las poblaciones de estos peces en su medio natural. El objetivo de este trabajo fue determinar la fidelidad al sitio y el rango de hogar del caballito de mar narizón (Hippocampus reidi Ginsburg, 1933), especie pobremente estudiada y clasificada en la categoría de Datos Deficientes (DD) por la UICN. Los muestreos se realizaron entre abril de 2009 y agosto de 2010 en dos estaciones localizadas en la dársena de Varadero, que es un canal artificial localizado al noroeste de Cuba. Se llevaron a cabo censos visuales en 17 transectos $(20 \times 1 \mathrm{~m})$ en cada estación. Un total de 68 individuos fueron identificados y marcados con elastómeros fluorescentes. $H$. reidi mostró fidelidad al sitio, recapturándose el $48.5 \%$ de los ejemplares marcados muy cerca o en el mismo transecto donde fueron localizados y censados por primera vez. El rango de hogar fue de $70.6 \pm 60.1 \mathrm{~m}$ (media \pm desviación estándar) y no se encontraron diferencias significativas en esta variable entre ambas estaciones o entre las hembras y los machos. Estos resultados incrementan el conocimiento actual que se tiene acerca de esta especie de caballito de mar y demuestran la vulnerabilidad de sus poblaciones a las posibles perturbaciones de su restringido hábitat.
\end{abstract}

Palabras claves: Hippocampus reidi, caballito de mar narizón, fidelidad al sitio, rango de hogar, Cuba.

\begin{abstract}
Fish populations of the Syngnathidae family, including the charismatic seahorse, have significantly declined worldwide during the last decades. Up to now, these populations have been poorly researched in their habitat in Cuba. The objective of this study was to determinate site fidelity and home range of the longsnout seahorse (Hippocampus reidi Ginsburg, 1933), which has been poorly studied and has been classified as a Data Deficient (DD) species by the IUCN. Sampling was conducted between April 2009 and August 2010 in two stations located at the dock of Varadero, an artificial channel located northwest of Cuba. Visual census were conducted on 17 transects $(20 \times 1 \mathrm{~m})$ at each station, and a total of 68 seahorses were identified and tagged with fluorescent elastomers. $H$. reidi showed site fidelity, with $48.5 \%$ of the marked individuals being recaptured close to or on the same transect where they were located the first time. The home range was 70.6 $\pm 60.1 \mathrm{~m}(\mathrm{mean} \pm \mathrm{SD})$, and no significant differences were found in this variable between both stations or between males and females. These results increase our current knowledge on this seahorse species and show the vulnerability of these populations to potential damages of their restricted habitat.
\end{abstract}

Keywords: Hippocampus reidi, longsnout seahorse, site fidelity, home range, Cuba.

\footnotetext{
1 Acuario Nacional de Cuba. Ministerio de Ciencia, Tecnología y Medio Ambiente. Ave. 1ra. No. 6024. Miramar. Playa. La Habana CP 11300 Cuba. darildelanuez@gmail.com*
}

Recibido: 8 de octubre de 2015

Corregido: 11 de enero de 2016

Aceptado: 13 de enero de 2016

DOI: http://dx.doi.org/10.15359/revmar.8-1.7 


\section{INTRODUCCIÓN}

La familia Syngnathidae se caracteriza porque sus representantes poseen formas del cuerpo muy peculiares e incluye peces tan carismáticos como los caballitos de mar, los dragones de mar y los peces pipa (Lourie et al. 2004). Sus peculiaridades biológicas, como la gestación llevada a cabo por los machos en una bolsa abdominal y en algunos casos la monogamia, proporcionan a la ciencia una oportunidad inusual en los estudios de ecología reproductiva. Además, son importantes depredadores de pequeños organismos planctónicos y de otros habitantes del fondo marino, por lo que su desaparición en un área podría perturbar el equilibrio de los ecosistemas costeros. Por otro lado, constituyen una relevante fuente de ingresos para los pescadores de subsistencia en muchos lugares del mundo. Estos y otros motivos han llevado a que estos peces, en especial los hipocampos, sean un foco fundamental de esfuerzos para su conservación (CITES, 2004; Giles et al. 2005).

En los últimos años, se ha observado un decline importante de la abundancia de individuos en muchas poblaciones de signátidos de todo el mundo, lo cual ha sido atribuido a diversas causas, como la pérdida de hábitat, las enfermedades, la presencia de especies invasoras, la pesca incidental $\mathrm{y} / \mathrm{o}$ directa, entre otras (Baum \& Vincent, 2005; MartinSmith \& Vincent, 2005). Además, algunas características, como la baja fecundidad y el escaso rango de movimientos de los peces de esta familia, contribuyen a incrementar el efecto que representan las amenazas previamente mencionadas (Foster \& Vincent, 2004).

A pesar de los riesgos a los que están sometidos, son pocos los estudios que se han realizado en el mundo para analizar características conductuales como son la fidelidad al sitio de asentamiento o el rango de hogar de las diferentes especies de signátidos. Estos conocimientos resultan necesarios para la creación de estrategias de manejo efectivas y programas de conservación in situ (Harasti et al. 2014). Para ello, se requiere de la identificación particular de los ejemplares, principalmente mediante el marcaje. Esta técnica facilita el seguimiento individualizado y de la población, permitiendo determinar patrones de movilidad, de la estructura de las poblaciones, etología, comportamiento reproductivo y de crecimiento, entre otros aspectos ecológicos importantes.

Aunque existen numerosas técnicas de marcaje con muy diferente grado de efectividad (Griffiths, 2002; Doupé et al. 2003), idealmente la técnica que se emplee debe afectar lo menos posible la conducta, la supervivencia, la reproducción o el crecimiento del animal en estudio, así como poseer altas tasas de retención que permitan obtener altos porcentajes de recapturas antes de deteriorarse (Woods \& Martin-Smith, 2004). En el caso de los signátidos, una de las técnicas de marcaje más utilizadas en los últimos años han sido los implantes de elastómeros, etiquetas subcutáneas 
empleadas con éxito en estudios ecológicos (Sánchez-Camara et al. 2006; Sogabe \& Yanagisawa, 2007; Harasti et al. 2010).

Independientemente del método empleado para el marcaje, la mayoría de las investigaciones han constatado que los signátidos adultos suelen presentar una alta fidelidad al sitio y un rango de hogar muy pequeño (Vincent \& Sadler, 1995; Sánchez-Camara \& Booth, 2004). Estas características incrementan la vulnerabilidad de sus poblaciones, ya que al usar espacios muy restringidos, cualquier perturbación del hábitat o extracción de individuos con fines comerciales puede representar un alto riesgo para su supervivencia.

Por estas razones, el género Hippocampus ha sido añadido en el Apéndice II de la Convención sobre el Comercio Internacional de Especies Amenazadas de Fauna y Flora Silvestres (CITES, 2004) y 33 de sus especies se encuentran en la Lista Roja de las especies amenazadas según la Unión Internacional para la Conservación de la Naturaleza (UICN, 2015). Por otro lado, la mayoría de ellas han sido incluidas en la categoría de Datos Deficientes (DD), lo cual refleja que los estudios realizados en el medio natural son todavía insuficientes.

Tal es el caso de las poblaciones de este grupo de peces de Cuba, donde hasta el momento los estudios enfocados a incrementar el conocimiento de la ecología de los caballitos de mar han sido escasos (Pastor et al. 2011), y no se ha realizado ninguno que analice el uso del espacio (fidelidad al sitio y rango de hogar) para ninguna de las especies presentes en sus aguas territoriales, a pesar de la importancia que esto representa para la conservación y el manejo de estas. El presente trabajo es el primer estudio de este tipo en Cuba y los esfuerzos se han centrado sobre el caballito de mar narizón, Hippocampus reidi Ginsburg, 1933.

Esta especie se distribuye en amplias zonas de la costa Atlántica de América, desde Carolina del Norte hasta Brasil, incluyendo el Mar Caribe y el Golfo de México (Lourie et al. 2004). Se encuentra habitualmente en zonas marinas y estuarinas someras, en profundidades que van desde apenas $10 \mathrm{~cm}$ (Rosa et al. 2002) hasta los 55 m (Vari, 1982) y se asocia a pastos marinos, arrecifes de coral, manglares y Sargassum, principalmente. El estado de sus poblaciones es muy poco conocido debido a la escasez de estudios hasta el momento, por lo que la UICN (2015) la coloca en la categoría de Datos Deficientes (DD). No obstante, se sabe que se trata de una especie que sufre una importante presión debido a la extracción por parte de acuaristas, para la medicina tradicional, con fines religiosos, como curiosidad, entre otros aspectos, por lo que se considera fuertemente amenazada (Rosa et al. 2002). Los resultados obtenidos en este estudio constituyen un valioso aporte en los esfuerzos para conocer más sobre su ecología y contribuirán al desarrollo de futuros planes de manejo y conservación en el país y en la región. 


\section{MATERIALES Y MÉTODOS}

\section{Sitio de muestreo}

Los datos se tomaron entre abril de 2009 y agosto de 2010 . No obstante, es el único estudio de este tipo efectuado hasta el momento en Cuba, por lo que cualquier información aportada sobre la ecología de esta especie posee un gran valor científico. Los muestreos se realizaron en dos estaciones localizadas en el interior de la dársena de Varadero, provincia de Matanzas, región noroccidental de Cuba (Fig. 1). Ambos puntos están separados aproximadamente 1600 metros uno del otro y fueron identificados como Barracuda (BR) y Playa Caleta (PC).

La dársena de Varadero es un canal artificial semicerrado con relativamente poca actividad náutica, con muelles y pilotes para el amarre de embarcaciones que se encuentran en malas condiciones estructurales y sobre los que se ha desarrollado un complejo ecosistema bentónico. Esta región geográfica está caracterizada por una marcada falta de estacionalidad, donde solo pueden establecerse con claridad una época de seca (entre noviembre y abril) y una de lluvias (entre mayo y octubre). La temperatura media del agua en la dársena durante el tiempo de estudio fue de 25.1 $\pm 0.4^{\circ} \mathrm{C}$. En el interior del canal existen corrientes de marea, pero los valores de estas y su periodicidad aún deben ser cuantificados en futuros estudios.

Barracuda (BR): Ubicada en los $23^{\circ} 08.04^{\prime} \mathrm{N}$ y $081^{\circ} 17.15^{\prime} \mathrm{W}$, es la localidad más cercana a las compuertas interiores del canal. Consta de un muelle de concreto que incluye una pared y varios pilotes con una profundidad de entre 1.0 y $1.5 \mathrm{~m}$. En

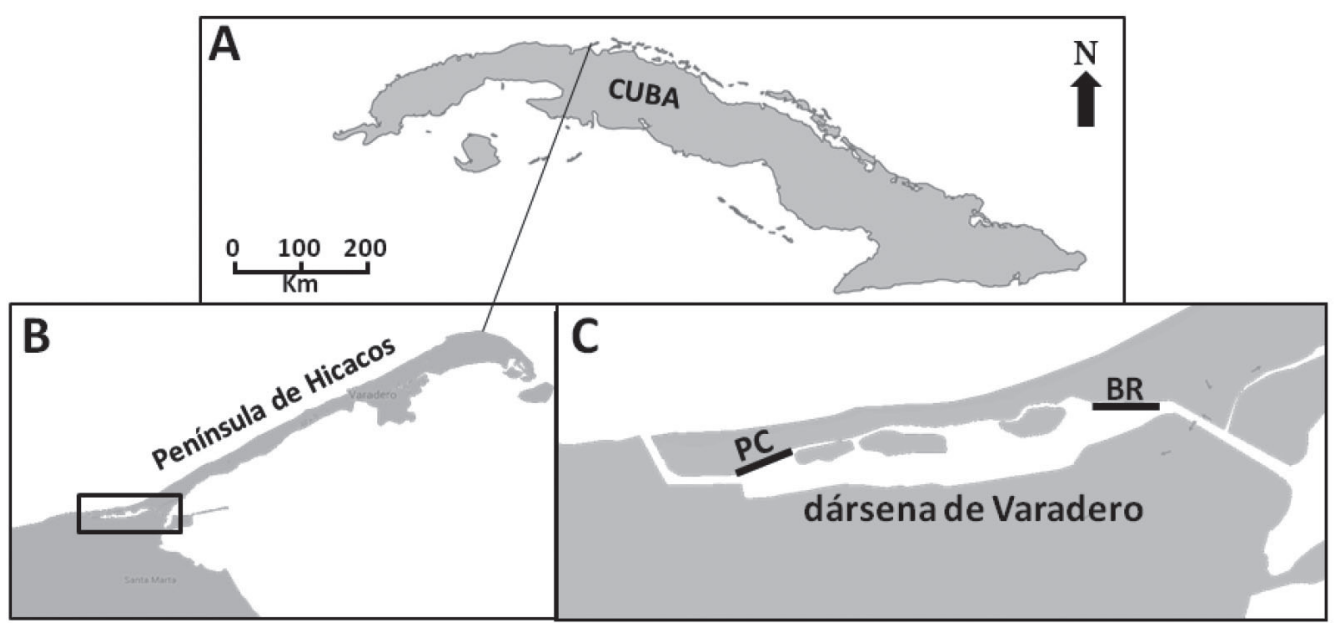

Fig. 1. Ubicación geográfica de la zona de estudio. (A) Isla de Cuba y localización de la Península de Hicacos. (B) Ubicación de la dársena de Varadero. (C) Estaciones de muestreo Barracuda (BR) y Playa Caleta (PC)

Fig. 1. Geographic location of research area. (A) Island of Cuba and location of the Península de Hicacos. (B) Location of the Varadero dock. (C) Barracuda (BR) and Playa Caleta (PC) sampling stations 
la pared existen desagües activos de aguas residuales, por lo que se observa en general una gran cantidad de partículas en suspensión. El fondo es mayormente fangoso y existe una gran cantidad de organismos bentónicos asociados a la pared y los pilotes, entre los que se destacan: algas de los géneros Ulva y Caulerpa, esponjas de los géneros Dysidea, Tedania y Halidona, bivalvos, poliquetos tubícolas (Sabellastarte magnifica) y ascidias, fundamentalmente.

Playa Caleta (PC): Situada en los $23^{\circ} 07.91^{\prime} \mathrm{N}$ y $081^{\circ} 18.25^{\prime} \mathrm{W}$. Consta de un muelle de concreto que incluye una pared y varios espigones con una profundidad que oscila entre 1.5 y 2.0 $\mathrm{m}$. Esta estación se encuentra más cerca de la salida del canal al mar abierto en la costa norte que la estación de BR, por lo que tiene más probabilidades de renovación del agua por las corrientes $y$ esto influye en que la visibilidad sea mayor. En esta estación no existe tanta abundancia de organismos bentónicos que cubren los espigones como en la estación de BR, aunque las especies que se encuentran son mayormente las mismas. El sustrato es fangoso-arenoso.

\section{Protocolo del estudio}

Se realizaron en total 17 viajes de muestreo, 10 en la estación de BR y 7 en la de PC. En cada estación se demarcaron 17 transectos lineales de 20 $\mathrm{m}$ de largo por $1 \mathrm{~m}$ de ancho y paralelos al margen del canal, cubriendo un área de $340 \mathrm{~m}^{2}$ en cada sitio. Los transectos, ubicados linealmente uno a continuación del otro, abarcaron la distancia total existente entre el inicio y el final de la zona de pilotes (toda la zona de muelles) y fueron exhaustivamente inspeccionados en censos visuales realizados mediante buceo libre por 3-4 colaboradores cada vez (Fig. 2).

Se marcaron un total de 68 ejemplares de $H$. reidi (43 en BR y 25 en PC) empleando elastómeros fluorescentes subcutáneos (VIE, North-west Marine Technologies, Inc. Shaw Island, $W A, U S A$ ) para su identificación individual. Este tipo de marcador consiste en un polímero sintético que, al ser inyectado debajo de la piel, se endurece sin provocar reacciones adversas y puede detectarse fácilmente ayudado por una fuente de luz ultravioleta (UV). Previo a la recolecta de los ejemplares, se preparó la marca utilizando combinaciones de los colores amarillo y rojo, los más fáciles de detectar según el patrón de coloración de los caballitos en esta

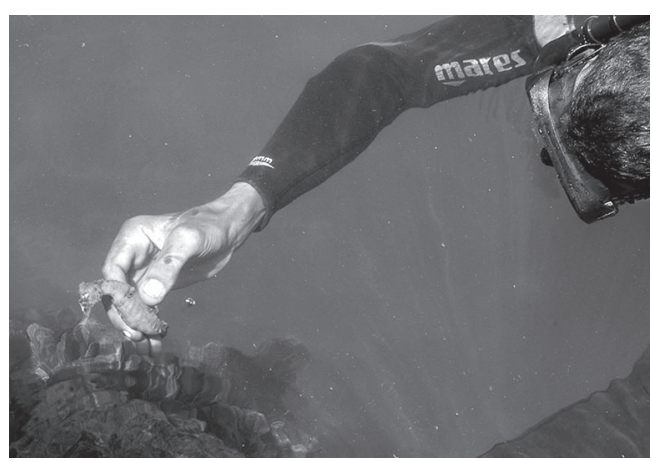

Fig. 2. Buceador capturando un ejemplar macho de Hippocampus reidi en la estación Barracuda (BR). Fotografía tomada el 23 de diciembre de 2009

Fig. 2. Diver capturing a male specimen of Hippocampus reidi in the Barracuda station (BR). Picture taken December 23rd, 2009 
zona. A todos los ejemplares se les determinó el sexo con facilidad, teniendo en cuenta que los machos poseen una bolsa incubadora muy evidente en su región abdominal y las hembras carecen de esta (Lourie et al. 2004).

Cada caballito se marcó con dos etiquetas VIE en los segmentos torácicos y de la cola debidamente numeradas de manera que por su posición y su color, resultaran en una combinación única que permitiera su posterior identificación. En el caso de los machos, solo se colocaron etiquetas en los segmentos torácicos, para evitar colocar el implante cerca de la bolsa incubadora (Fig. 3). El código de la marca se registró en una planilla, así como la presencia de algún carácter morfológico distintivo que facilitara la identificación del ejemplar en caso de pérdida o deterioro de la etiqueta. Una vez marcados, los caballitos fueron liberados en el sitio exacto donde se encontraban al capturarlos.

\section{Análisis de los datos}

La fidelidad al sitio y el rango de movimientos se determinaron siguiendo el criterio de Sánchez-Camara \& Booth (2004) como se describe a continuación.

Fidelidad al sitio: Se determinó a partir de la persistencia de los caballitos (porcentaje de recapturas) en el sitio donde fueron originalmente marcados. Las recapturas realizadas durante el período de estudio se dividieron en bloques de tres meses, con el objetivo de agrupar un mayor número de reavistamientos de ejemplares.

Rango de hogar: Se determinó por la distancia total (en metros), medida

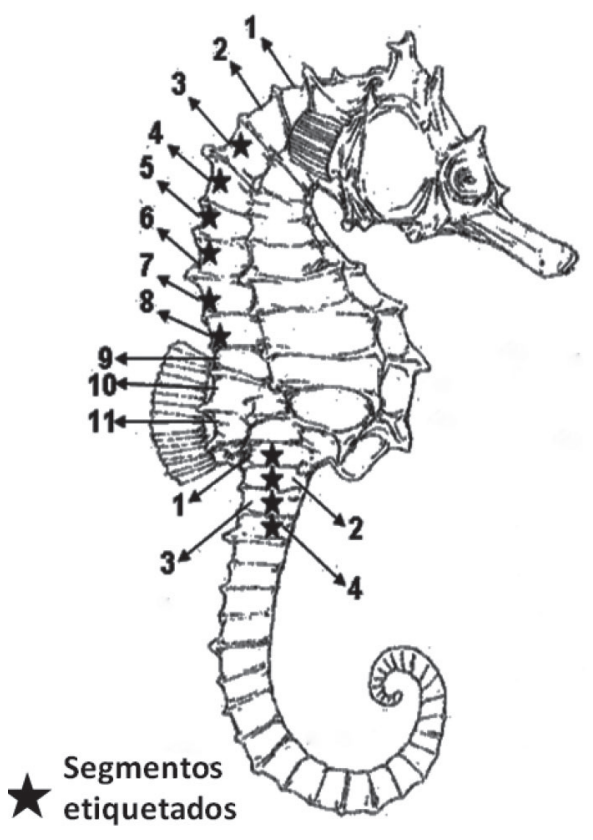

Fig. 3. Numeración de los segmentos torácicos (3-8) y de la cola (1-4) donde se colocaron implantes de elastómeros fluorescentes en Hippocampus reidi para su identificación

Fig. 3. Numbering of back (3-8) and tail (14) segments where fluorescent elastomer tags were placed for the individual identification of Hippocampus reidi

entre los extremos donde fue encontrado cada individuo durante el período de estudio. Debido a que los datos no siguieron una distribución normal, los rangos de hogar entre hembras y machos en cada sitio y entre los caballitos de ambas estaciones fueron comparados empleando la prueba pareada no paramétrica de comparación de medias de Mann-Whitney considerando significativas las diferencias para $P$ $<0.05$, con intervalos de confianza al $95 \%$. Se empleó el programa Statistica 


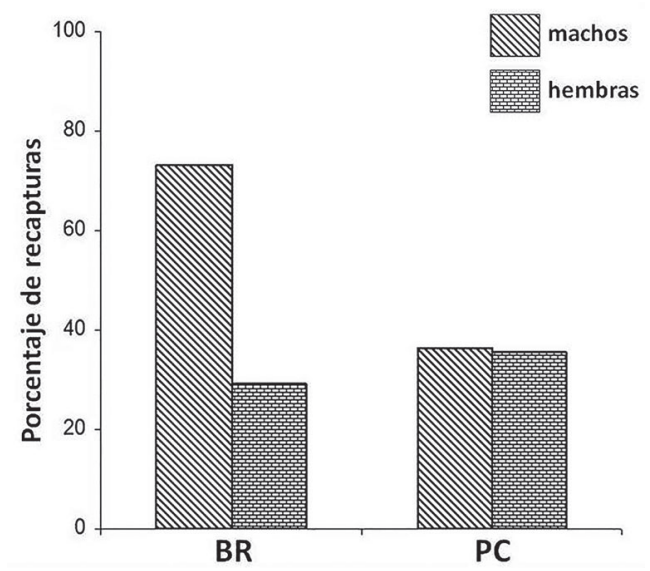

Fig. 4. Porcentaje de ejemplares de Hippocampus reidi recapturados en Barracuda (BR) y Playa Caleta (PC) por sexo durante el período de estudio

Fig. 4. Percentage of seahorses Hippocampus reidi by sex recaptured in Barracuda (BR) and Playa Caleta (PC) during the research period

8.0 para los análisis correspondientes (StatSoft, 2007).

\section{RESULTADOS}

\section{Fidelidad al sitio}

De los 68 ejemplares de $H$. reidi marcados, 33 fueron recapturados al menos una vez durante el período de estudio, lo que representa un $48.5 \%$ de recapturas entre ambas localidades. Por estaciones, en BR, de los 43 ejemplares que se marcaron, fueron recapturados $24(55.4 \%)$, mientras que en PC fueron recapturados 9 de un total de 25 (36\%). Cuando se analizó por sexo, se obtuvo que en BR fueron recapturados al menos una vez 19 machos de los 26 etiquetados $(73.1 \%)$ y 5 hembras de las 17 etiquetadas (29.4\%). En PC, donde se capturaron y marcaron menos ejemplares, fueron recapturados 4 de los 11 machos marcados (36.4\%) y 5 de las 14 hembras (35.7\%) (Fig. 4).

En ambos sitios, el mayor número de caballitos de mar se recapturó en el primer trimestre del año a partir del momento en que fueron marcados, a partir del segundo trimestre las recapturas fueron disminuyendo, aunque en el cuarto trimestre aún fueron encontrados ejemplares marcados. En el caso particular de $\mathrm{BR}$, en todos los trimestres predominó la recaptura de los ejemplares machos. Sin embargo, en PC la recaptura por sexos se comportó de manera muy similar en el primer y el último trimestres, ya que en el segundo no se recapturaron ejemplares y en el tercer trimestre solo se recapturó una hembra (Fig. 5).

\section{Rango de hogar}

Excepto un ejemplar hembra, que fue capturado y etiquetado en PC y recapturado en $\mathrm{BR}$, el resto de los ejemplares fueron recapturados en la misma estación donde fueron avistados por primera vez, y en muchos casos, muy cerca o incluso en el mismo transecto donde fueron etiquetados (Fig. 6). Por ser una excepción notable, para el análisis estadístico se excluyó solo ese individuo que se movió aproximadamente $1820 \mathrm{~m}$.

Teniendo en cuenta la totalidad de ejemplares recapturados entre ambos sitios, el rango de hogar (media \pm desviación estándar) de $H$. reidi en la dársena de Varadero fue de $70.6 \pm 60.1$ $\mathrm{m}$. Por estación se determinó que el rango para $\mathrm{BR}$ fue de $56.6 \pm 44.0 \mathrm{~m}$ y 

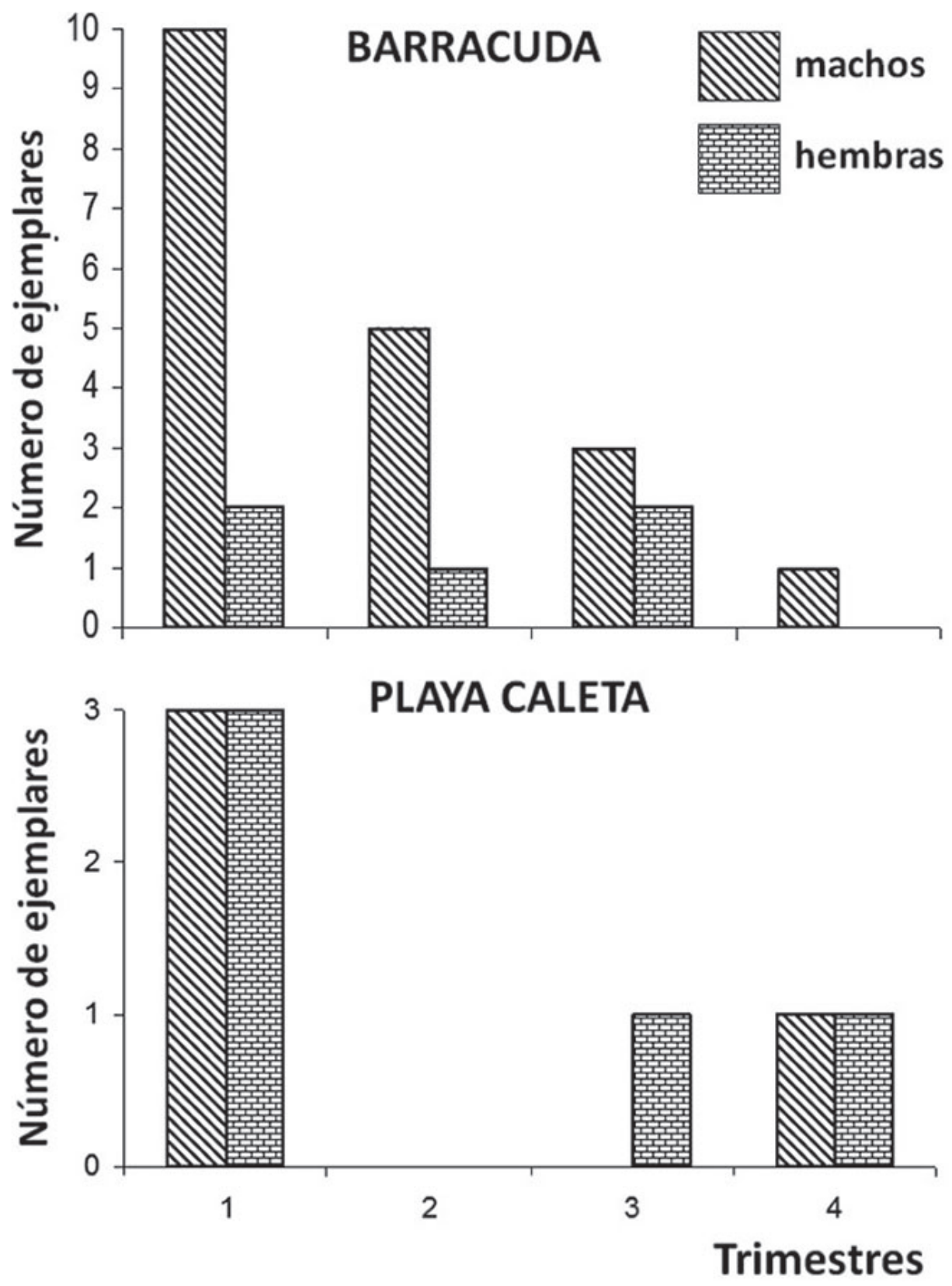

Fig. 5. Número de ejemplares de Hippocampus reidi recapturados por trimestre por sexo en cada estación

Fig. 5. Number of seahorses Hippocampus reidi by sex recaptured in 3-month periods in each sampling station 
en PC fue de $112.5 \pm 83.5 \mathrm{~m}$. Al analizar esta variable por sexos, se obtuvo que en BR el rango de hogar de los machos fue de $50.5 \pm 35.5 \mathrm{~m}$, mientras que el de las hembras fue de $80.0 \pm 67.8 \mathrm{~m}$. En PC para los machos fue de $110 \pm$ $80.8 \mathrm{~m}$ y para las hembras fue de $115 \pm$ $98.5 \mathrm{~m}$. Los altos valores de dispersión obtenidos con respecto a la media son un indicador de la variabilidad encontrada en los datos recogidos, pues mientras muchos individuos permanecieron prácticamente durante todo el tiempo de estudio en el mismo transecto donde fueron marcados o muy cerca de este, otros se desplazaron distancias mayores (Fig. 6). En este sentido, en ambos sexos la distancia máxima que se movieron dentro de cada estación fue de alrededor de $200 \mathrm{~m}$, un desplazamiento llamativo tratándose de una especie pequeña y de movilidad anatómica reducida.

No se encontraron diferencias significativas en el rango de hogar de la especie entre ambas estaciones, así como tampoco entre los machos y las hembras en $\mathrm{BR}$ ni en $\mathrm{PC}$. Al comparar el rango de hogar de los machos entre ambas estaciones, tampoco se encontraron diferencias significativas, obteniéndose igual resultado al comparar esta variable entre las hembras (Fig. 7).

\section{DISCUSIÓN}

Los resultados de este estudio nos confirman aspectos de la conducta de $H$. reidi previamente comprobados en otras especies de signátidos, y en particular de caballitos de mar. Los porcentajes de recaptura obtenidos, en especial los de la estación de BR, sugieren que los adultos de $H$. reidi presentan fidelidad al sitio, similar a lo reportado por Rosa et al. (2007) para esta misma especie en Brasil, Curtis \& Vincent (2006) para H. guttulatus en Portugal, así como Moreau \& Vincent (2004) en $H$. breviceps y Harasti et al. (2014) para $H$. whitei en Australia. Una marcada fidelidad también ha sido comprobada en el pariente cercano de los caballitos de mar Phyllopteryx taeniolatus, un dragón de mar habitante de las costas australianas (SánchezCamara \& Booth, 2004).

El uso de un área preferencial, y por lo tanto la fidelidad a esta por los adultos de caballitos de mar, puede estar relacionado con las facilidades que el sitio les ofrece para alimentarse o como área de refugio y descanso (Harasti et al. 2012). Estas características las reúnen sobre todo hábitats donde abundan las algas, las plantas marinas y la fauna sésil, como las esponjas o los corales blandos. Aunque no está suficientemente estudiado, se estima que los caballitos de mar tienen preferencias por estos entornos de estructura compleja donde se facilita mucho el camuflaje y la posibilidad de emboscar a sus presas (Flynn \& Ritz, 1999). Esto influiría de forma notable en la fidelidad al sitio de estos peces de poca capacidad para nadar con rapidez (Kleiber et al.2011).

En BR pudimos observar que el hábitat era bastante más complejo que en PC, pues la superficie de los espigones estaba cubierta prácticamente 

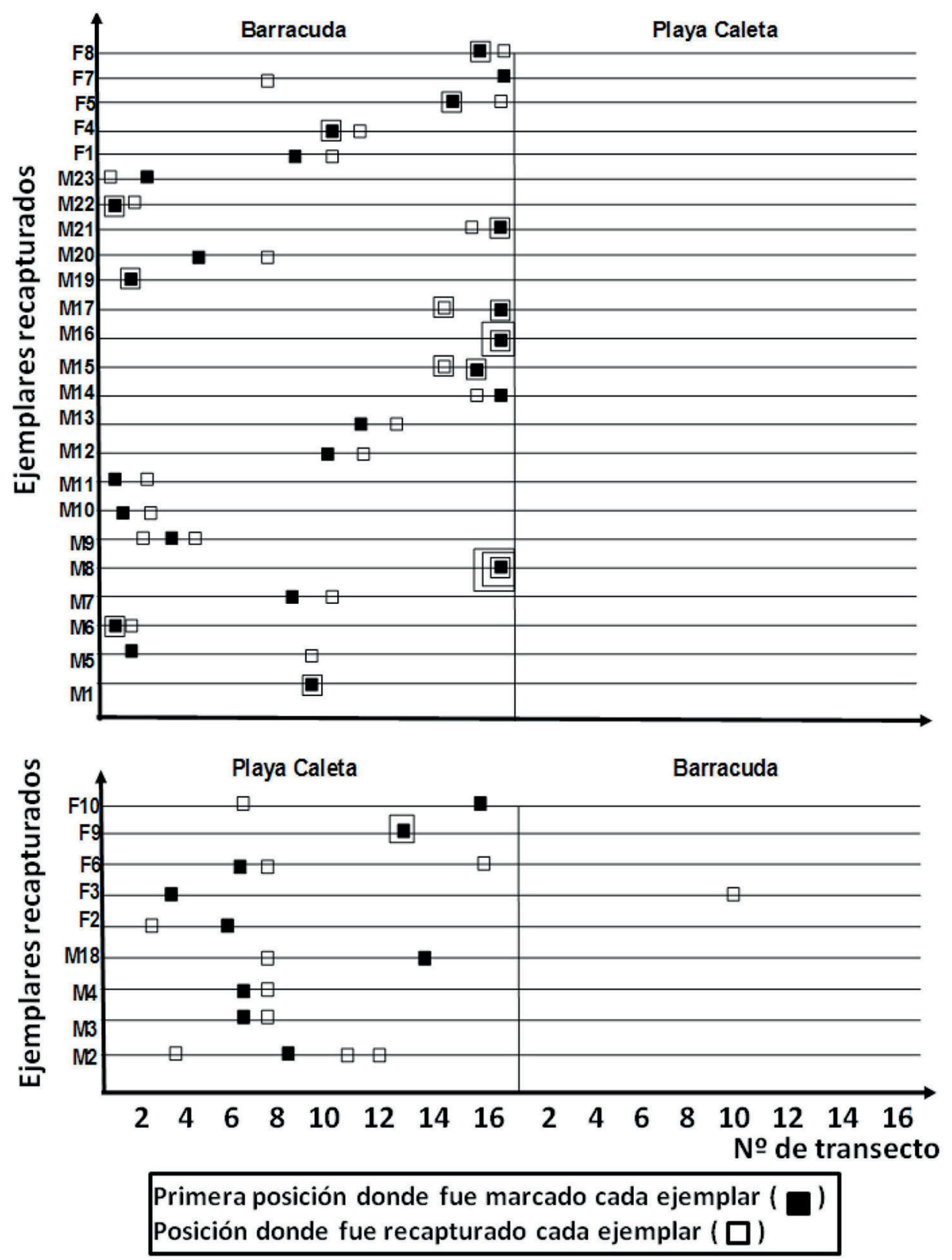

Fig. 6. Transectos donde fueron recapturados los ejemplares machos (M) y hembras (F) de Hippocampus reidi durante el período de estudio en cada estación de muestreo. Los cuadros solapados indican que las recapturas se realizaron en el mismo transecto Fig. 6. Transects where the Hippocampus reidi, both male (M) and female (F), were recaptured during the research period in each sampling station. The overlapping boxes indicate that re-catches were made along the same transect 
en su totalidad de organismos sésiles, en particular algas, esponjas y bivalvos, que potenciaban la capacidad críptica y de anclaje de $H$. reidi. En cambio, en PC la superficie de los espigones presentaba frecuentes áreas desprovistas de algas y fauna bentónica, por lo que los caballitos eran detectados con más facilidad por los buceadores que colaboraron en este estudio. Rosa et al. (2007) y Mai \& Rosa (2009) registraron un uso frecuente de las macroalgas, las raíces de mangle, los cnidarios, las esponjas, las plantas marinas y los briozoos como medios de sujeción de esta especie, aunque estudios cuantitativos acerca del empleo de estos potenciales sustratos por parte de $H$. reidi aún deben ser realizados. Por otro lado, Hellyer et al. (2011) determinaron que ciertos microcrustáceos, como los anfipodos, son significativamente más abundantes en aquellos sitios de mayor complejidad estructural, y estos constituyen una parte de la dieta de los caballitos de mar según Kuiter (2009), incluido H. reidi (Felício et al. 2006). Las potenciales características más favorables observadas en este hábitat podrían apoyar la explicación de que los porcentajes de recaptura en $\mathrm{BR}$ fueran mayores que en $\mathrm{PC}$, donde por el contrario, el entorno no fue tan complejo. En este sentido, los caballitos podrían estar más expuestos a la depredación y/o las capturas ilegales al ser más fácilmente detectables.

No se descarta que exista una pequeña posibilidad de que los porcentajes de recaptura obtenidos en este estudio fueran afectados debido a un aumento de la depredación de los caballitos de mar como consecuencia de la etiqueta, que podría disminuir su capacidad críptica. Aunque pocos trabajos han abordado este tema hasta hoy, un estudio realizado por Malone et al. (1999) en dos especies de gobios demostró que el marcaje no afectó la vulnerabilidad de estos peces a la depredación. Lo mismo constataron Harasti et al. (2012) en $H$. whitei, quienes pudieron observar ejemplares vivos hasta después de cuatro años de haber sido marcados con elastómeros por primera vez.

La visibilidad de la etiqueta VIE disminuye considerablemente, aunque de forma gradual a medida que transcurre el tiempo. Esto se debe sobre todo al crecimiento de tejido pigmentado en la piel situada sobre esta. De esta manera, aunque los porcentajes de retención usuales del elastómero son altos (hasta más de un año después de su colocación), las marcas solo pueden ser detectadas con facilidad mediante el uso de una linterna de luz ultravioleta (Olsen \& Vøllestad, 2001; Curtis, 2006; Josephson et al. 2008). Esto, unido al pequeño tamaño de la etiqueta, quizás no implicaría efectos negativos en la capacidad críptica de los caballitos, aunque estudios más profundos encaminados a determinar los potenciales efectos se deben realizar. No obstante, debemos destacar que durante los muestreos detectamos la presencia creciente de peces león (Pterois sp.) dentro de la dársena, una voraz especie invasora que en el 2009 había ocupado ya una gran parte de las 

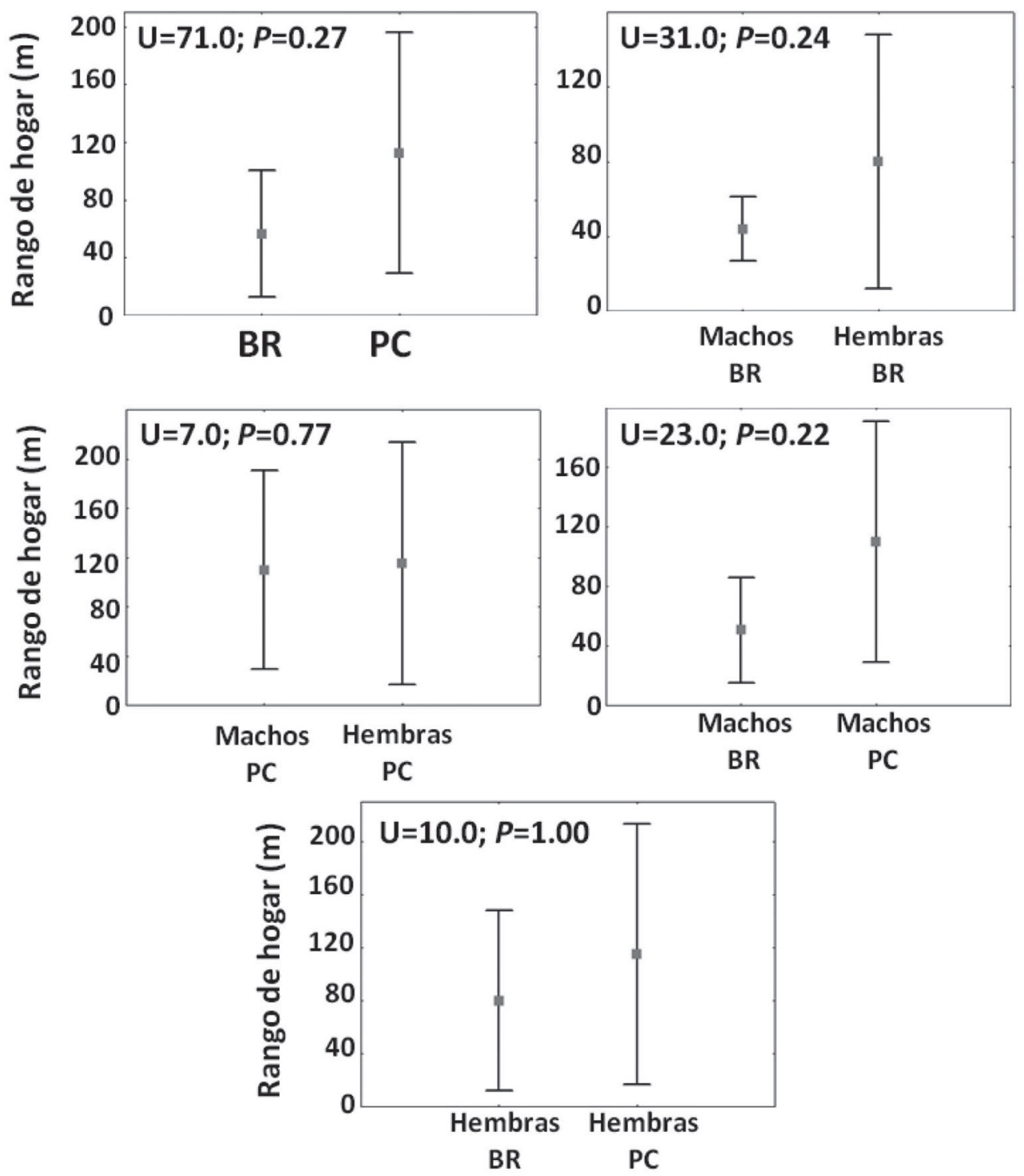

Fig. 7. Rango de hogar (media y desviación estándar) de Hippocampus reidi en las estaciones de Barracuda (BR) y Playa Caleta (PC). Las letras corresponden al valor de la prueba "U" de Mann-Whitney y el valor de P (no significativo en todos los casos) Fig. 7. Home range (mean and standard deviation) of Hippocampus reidi in the Barracuda (BR) and Playa Caleta (PC) sampling stations. The letters indicate the Mann-Whitney test results (letter " $U$ ") and P values (not significant in all cases) 
zonas marinas del archipiélago cubano (Chevalier et al. 2014) y cuyos efectos sobre las especies nativas, incluidos los caballitos de mar, se están aún por evaluar a mediano y largo plazos.

En nuestro estudio encontramos que el rango de hogar para $H$. reidi fue relativamente pequeño y en la mayoría de los ejemplares las recapturas ocurrieron en el mismo transecto donde fueron marcados al inicio o muy cerca de este. Vincent et al. (2004) y Curtis \& Vincent (2006) también reportan rangos de hogar pequeños para las especies $H$. whitei $\left(14.4 \pm 3.9 \mathrm{~m}^{2}\right.$ para los machos y 9.0 $\pm 3.2 \mathrm{~m}^{2}$ para las hembras) e $H$. guttulatus $\left(19.9 \pm 12.4 \mathrm{~m}^{2}\right)$. En ocasiones pudimos detectar corrientes de marea relativamente fuertes en ambas estaciones de muestreo. Este factor podría influir en el rango de hogar obtenido, ya que, aunque estos peces tienen mecanismos eficaces para sostenerse al sustrato, en ocasiones podrían verse arrastrados varios metros del sitio donde fueron localizados.

Según Foster \& Vincent (2004), los hipocampos mantienen un rango de hogar pequeño quizás porque el costo del movimiento para estas especies es mayor que el beneficio que obtienen de él en términos de supervivencia, crecimiento y reproducción. Movimientos más amplios podrían comprometer la estrategia de camuflaje con el medio, ya que los cambios de coloración generalmente en estas especies no son tan rápidos, y en las especies que lo hacen de forma más inmediata, como $H$. whitei según refieren Vincent et al. (2005), o especies de otros taxones como pulpos y sepias (por ejemplo), estos largos desplazamientos podrían llamar la atención de los depredadores y las potenciales presas.

Aunque en nuestro estudio los valores medios absolutos del rango de hogar fueron ligeramente mayores para las hembras, no se encontraron diferencias significativas entre ambos sexos, coincidiendo con lo reportado por Perante et al. (2002) para la especie H. comes y Caldwell \& Vincent (2013) para $H$. guttulatus. Sin embargo, en un estudio realizado previamente para $H$. reidi por Dauwe (1993) en la isla de Bonaire, las hembras sí mostraron un rango de hogar mayor que los machos, por lo que se sugiere incrementar el número de localidades de estudio que abarquen una mayor extensión dentro de su área de distribución geográfica, para determinar cuál de los dos patrones resulta con mayor frecuencia.

Moreau \& Vincent (2004) también observaron que en las costas de Victoria, Australia, las hembras de H. breviceps presentaron rangos de movilidad más amplios en comparación con los machos. Resultados similares fueron encontrados por Harasti et al. (2014) en la región australiana de Nueva Gales del Sur para $H$. whitei. Aunque nuestros resultados no muestran diferencias entre los sexos en este sentido, Vincent et al. (2005) han planteado la hipóte- 
sis de que los machos de caballitos de mar podrían presentar una capacidad de desplazamiento más limitada debido a la necesidad de conservar energía en la etapa reproductiva, ya que son ellos los responsables de portar los embriones en su bolsa. Este saco abdominal, que en algunas ocasiones puede ser bastante voluminoso, podría entorpecer también sus movimientos e incrementar la masa de arrastre al nadar, motivando una menor movilidad de los machos durante esta época. En Cuba no se ha estudiado aún cuál es el período reproductivo de esta especie, pero a modo anecdótico podemos decir que identificamos machos que portan embriones en su bolsa durante todo el año, por lo que aparentemente no existe una época reproductiva como tal, sino que lo hacen de forma permanente, tal vez debido a la ausencia de estacionalidad, lo cual coincide con lo reportado para esta especie por Rosa et al. (2007) y Freret-Meurer \& Andreata (2008) en Brasil.

La ausencia de estaciones marcadas en Cuba, donde solo puede establecerse unaépocadeseca(entrenoviembrey abril) y una de lluvias (entre mayo y octubre), podría indicar que las variaciones de temperatura en el interior del canal no serían un factor muy importante no solo para la reproducción de estos peces, sino también para sus patrones de movimiento dentro de la dársena. No obstante, sería interesante comprobar en futuros estudios si pequeños cambios anuales en los valores de esta variable influyen de alguna manera en los movimientos de $H$. reidi en aguas someras.
La fidelidad al sitio y el pequeño rango de hogar que ha mostrado tener $H$. reidi en este estudio hacen que esta especie sea muy vulnerable a la explotación y la perturbación de su hábitat. Estas características conductuales, además de la bajísima densidad de población de la especie en Cuba (Pastor et al. 2011), implican que cualquier extracción o degradación del hábitat pueden afectar irremediablemente a la población. Con respecto al área de estudio, al ser un canal artificial y no un espacio natural, los riesgos de afectación de las poblaciones de $H$. reidi son más altos debido a las acciones previstas para el mantenimiento de la dársena de Varadero, como son el dragado del sustrato o la rehabilitación de los espigones como consecuencia del previsible incremento de la actividad náutica en los próximos años. Estas actividades podrían ser catastróficas para $H$. reidi en este sitio, donde se encuentra "la mayor densidad registrada para esta especie hasta el momento en Cuba" (L. Pastor, comunicación personal, septiembre 2, 2015). Es necesario, entonces, ampliar este estudio a otras zonas de la dársena y evaluar la dinámica de las poblaciones de $H$. reidi ante las presiones antropogénicas a las que se ven sometidas. Asimismo, elaborar planes y mecanismos que puedan contribuir a su protección, a la par del desarrollo socioeconómico de esta conocida zona turística de la isla mayor del archipiélago cubano. 


\section{CONCLUSIONES}

Según los resultados obtenidos, podemos concluir que en la dársena de Varadero Hippocampus reidi muestra alta fidelidad al sitio, así como un rango de hogar pequeño, características muy comunes a otras especies de la familia Syngnathidae y en particular a los caballitos de mar. El rango de hogar no mostró diferencias significativas entre las dos estaciones seleccionadas y tampoco se detectaron diferencias en el uso del espacio entre las hembras y los machos. Esto nos permite confirmar que se trata de una especie altamente vulnerable a la perturbación de su hábitat, y plantea la necesidad de ampliar estos estudios a otras regiones del país para analizar el comportamiento de la especie en zonas con características ecológicas diferentes.

\section{BIBLIOGRAFÍA}

Baum, J. K. \& Vincent, A. C. J. (2005). Magnitude and inferred impacts of the seahorse trade in Latin America. Environ. Conserv., 32(4), 305-319. doi: $10.1017 / \mathrm{S} 0376892905002481$

Caldwell, I. \& Vincent, A. J. (2013). A sedentary fish on the move: effects of displacement on long-snouted seahorse (Hippocampus guttulatus Cuvier) movement and habitat use. Environ. Biol. Fish., 96, 67-75. doi: 10.1007/ s10641-012-0023-4

Chevalier, P. P., Cabrera, E., Caballero, H., Corrada, R. I., Fernández, A., Cobián, D. \& García, A. (2014). Distribución, Abundancia y Relaciones Ecológicas del Pez León (Pterois volitans/miles: Scorpaenidae) en Cuba. GCFI, 66, 178-179.
CITES. (2004). Convención sobre el Comercio Internacional de Especies Amenazadas de Fauna y Flora Silvestres. Recuperado en agosto 29, 2015, disponible en http://www.cites.org/

Curtis, J. (2006). Visible implant elastomer color determination, tag visibility, and tag loss: Potential sources of error for marks-recapture studies. $N$. Am. J. Fish. Manag., 26, 327-337. doi: 10.1577/M05-099.1

Curtis, J. M. \& Vincent, A. C. (2006). Life history of an unusual marine fish: survival, growth and movementpatterns of Hippocampus guttulatus Cuvier 1829. J. Fish Biol., 68, 707-733. doi: 10.1111/j.0022-1112.2006.00952.x

Dauwe, B. (1993). Ecology of the Seahorse H. reidi (Syngnathidae) on Coral Reef of Bonaire (N. A.): Habitat, Reproduction and Community Interactions. Tesis de doctorado no publicada, Rijksuniversiteit Groningen, Groningen, Holanda.

Doupé, R. G., Partridge, G. J. \& Lymbery, A. J. (2003). Visible implant fluorescent elastomer tags as pedigree markers for applied aquaculture: an evaluation using black bream Acanthopagrus butcheri. Aquac. Res., 34(8), 681-683. doi: 10.1046/j.1365-2109.2003.00860.x

Felício, A. K. C., Rosa, I. L., Souto, A. \& Freitas, R. H. A. (2006). Feeding behavior of the longsnout seahorse Hippocampus reidi Ginsburg, 1933. J. Ethol., 24, 219-225. doi: 10.1007/ s10164-005-0189-8

Flynn, A. J. \& Ritz, D. A. (1999). Effect of habitat complexity and predatory style on the capture success of fish feeding on aggregated prey. J. Mar. Biol. Assoc. UK, 79, 487-494. doi: 10.1017/ S0025315498000617 
Foster, S. J. \& Vincent, A. C. (2004). Life history and ecology of seahorses: implications for conservation and management.J.Fish Biol., 65, 1-61.doi: 10.1111/j.0022-1112.2004.00429.x

Freret-Meurer, N. V. \& Andreata, J. V. (2008). Field Studies of a Brazilian Seahorse Population, Hippocampus reidi Ginsburg, 1933. Braz. Arch. Biol. Technol., 51(4), 743-751. doi: 10.1590/ S1516-89132008000400012

Giles, B. G., Ky, T. S., Hoang, D. H. \& Vincent, A. C. (2005). The catch and trade of seahorses in Vietnam. Biod. Conserv., 15, 2497-2513. doi: 10.1007/ s10531-005-2432-6

Griffiths, S. P. (2002). Retention of visible implant tags in small rockpool fishes. Mar. Ecol. Prog. Ser., 236, 307-309. doi: 10.3354/meps 236307

Harasti, D., Glasby, T. M. \& MartinSmith, K. M. (2010). Striking a balance between retaining populations of protected seahorses and maintaining swimming nets. Aquat. Conserv., 20, 159-166. doi: 10.1002/aqc.1066

Harasti, D., Gladstone, W. \& Martin Smith, K. M. (2014). Ontogenetic and sexbased differences in habitat preferences and site fidelity of the White's seahorse Hippocampus whitei. J. Fish Biol., 85, 1413-1428. doi: 10.1111/jfb.12492

Harasti, D., Martin-Smith, K. \& Gladstone, W. (2012). Population dynamics and life history of a geographically restricted seahorse, Hippocampus whitei.J. Fish Biol., 81, 1297-1314. doi: 10.1111/j.1095-8649.2012.03406.x

Hellyer, C. B., Harasti, D. \& Poore, A. G. B. (2011). Manipulating artificial habitats to benefit seahorses in SydneyHarbour. Aquat. Conserv., 21, 582-589. doi: 10.1002/aqc.1217
Josephson, D., Robinson, J. M., Weidel, B. C. \& Kraft, C. E. (2008). Long-Term Retention and Visibility of Visible Implant Elastomer Tags in Brook Trout. N. Am. J. Fish. Manag., 28, 1758-1761. doi: 10.1577/M08-019.1

Kleiber, D., Blight, L. K., Caldwell, I. R. \& Vincent, A. C. J. (2011). The importance of seahorses and pipefishes in the diet of marine animals. Rev. Fish Biol. Fisher, 21, 205-223. doi: 10.1007/s11160-010-9167-5

Kuiter, R. H. (2009). Seahorses and their relatives. Seaford, Australia: Aquatic Photographics.

Lourie, S. A., Foster, S. J., Cooper, E. W. T. \& Vincent, A. C. J. (2004). A Guide to the Identification of Seahorses. Washington, D.C., EE. UU.: University of British Columbia and World Wildlife Fund.

Mai, A. C. G. \& Rosa, I. M. L. (2009). Aspectos ecológicos do cavalomarinho Hippocampus reidi no estuário Camurupim/Cardoso, Piauí, Brasil, fornecendo subsídios para a criação de uma Área de Proteção Integral. Biota Neotrop., 9(3), 85-91. doi: 10.1590/ S1676-06032009000300007

Malone, J. C., Forrester, G. E \& Steele, M. A. (1999). Effects of subcutaneous microtags on the growth, survival, and vulnerability to predation of small reef fishes. J. Exp. Mar. Biol. Ecol., 237, 243-253. doi: 10.1016/ S0022-0981(99)00003-9

Martin-Smith, K. M. \& Vincent, A. C. (2005). Seahorse declines in the Derwent estuary, Tasmania in the absence of fishing pressure. Biol. Conserv., 123, 533-545. doi: 10.1016/j. biocon.2005.01.003 
Moreau, M. A. \& Vincent, A. C. (2004). Social structure and space use in a wild population of the Australian short-headed seahorse Hippocampus breviceps Peters, 1869. Mar. Freshwater Res., 55, 231239. doi: 10.1071/MF03159

Olsen, E. M. \& Vøllestad, L. A. (2001). An evaluation of visible implant elastomer for marking age- 0 brown trout. $N$. Am. J. Fish. Manag., 21, 967-970. doi: 10.1577/1548-8675(2001)021<0967:AE OVIE $>2.0 . \mathrm{CO} ; 2$

Pastor, L., Piloto, Y., Corrada, R. I. \& Chevalier, P. P. (2011). Estudio de las poblaciones de caballitos de mar en dos zonas de la costa norte de La Habana y Pinar del Río, Cuba. Rev. Mar. Cost., 3, 171-181.

Perante, N., Pajaro, M., Meeuwig, J. \& Vincent, A. (2002). Biology of a seahorse species, Hippocampus comes in the central Philippines. J. Fish Biol., 60, 821-837. doi: 10.1111/j.10958649.2002.tb02412.x

Rosa, I. L., Dias, T. L. \& Baum, J. K. (2002). Threatened fishes of the world: Hippocampus reidi Ginsburg, 1933 (Syngnathidae).Environ.Biol.Fish.,64, 378. doi: 10.1023/A:1016152528847

Rosa, I. L., Oliveira, T. P. R., Castro, A. L. C., Moraes, L., Xavier, J. H. A., Nottingham, M. C., ... \& Monteiro-Neto, C. (2007). Population characteristics, space use and habitat associations of the seahorse Hippocampus reidi (Teleostei: Syngnathidae). Neotrop. Ichthyol., 5, 405-414. doi: 10.1590/ S1679-62252007000300020

Sánchez-Camara, J. \& Booth, D. J. (2004). Movement, home range and site fidelity of the weedy seadragon Phyllopteryx taeniolatus (Teleostei: Syngnathidae).
Environ. Biol. Fish., 70, 31-41. doi: 10.1023/B:EBFI.0000022850.33643.e3

Sánchez-Camara, J., Booth, D. J., Murdoch, E. J., Watts, D. \& Turon, X. (2006). Density, habitat use and behaviour of the weedy seadragon Phyllopteryx taeniolatus (Teleostei: Syngnathidae) around Sydney, New SouthWales, Australia. Mar. Freshwater Res., 57, 737-745. doi: 10.1071/MF05220

Sogabe, A. \& Yanagisawa, Y. (2007). Sex-role reversal of a monogamous pipefish without higher potential reproductive rate in females. Proc. Biol. Sci., 274(1628), 2959-2963. doi: 10.1098/rspb.2007.1041

StatSoft, Inc. (2007). STATISTICA (data analysis software system), version 8 . www. statsoft.com. Tulsa, OK: StatSoft, Inc.

UICN. (2015). Red List of Threatened Species. Recuperado en septiembre 1, 2015, disponible en http://www.iucnredlist.org/

Vari, R. P. (1982). The seahorses (Subfamily Hippocampinae). In J. M. Böhlke, D. M. Cohen, B. B. Collette, W. N. Eschmeyer, R. H. Gibbs-Jr, T. W. Pietsch, W. J. Richards, C. L. Smith \& K. S. Thomson (Eds.), Fishes of the Western North Atlantic, Part 8. Order Gasterosteiformes, Suborder Syngnathoidea. Syngnathidae (Doryrhamphinae, Syngnathidae, Hippocampinae) (pp. 173-189). New Haven, EE. UU.: Sears Foundation for Marine Research.

Vincent, A. C., Marsden, A. D., Evans, K. L.\& Sadler, L. M. (2004). Temporal and spatial opportunities for polygamy in a monogamous seahorse, Hippocampus whitei. Behaviour, 141, 141-156. doi: $10.1163 / 156853904322890780$

Vincent, A. C. J., Evans, K. L. \& Marsden, A. D. (2005). Home range behaviour of the monogamous Australian seahorse, 
Hippocampus whitei. Environ. Biol. Fish., 72, 1-12. doi: 10.1007/s10641-004-4192-7 Vincent, A. C. \& Sadler, L. M. (1995). Faithful pair bonds in wild seahorses, Hippocampus whitei. Anim. Behav., 50, 1557-1569. doi: 10.1016/0003-3472(95)80011-5
Woods, C. \& Martin-Smith, K. M. (2004). Visible implant fluorescent elastomer tagging of the big-bellied seahorse, Hippocampus abdominalis. Fish. Res., 66, 363-371. doi: 10.1016/ $\mathrm{S} 0165-7836(03) 00183-8$ 